Pacific Journal of Mathematics

EXTENSIONS OF COMPLETELY REGULAR ORDERED 


\title{
EXTENSIONS OF COMPLETELY REGULAR ORDERED SPACES
}

\author{
T. H. Choe And Y. H. Hong
}

\begin{abstract}
In this paper we introduce the concept of $o$-completely regular filters on a completely regular ordered space in order to characterize compact ordered spaces and show that for any completely regular ordered space $X$, Nachbin compactification of $X$ is precisely the strict extension of $X$ with all maximal $o$-completely regular filters as the filter trace. With this concept, we also define $k$-compact ordered spaces for every infinite cardinal $k$, which give rise to a chain of extensive subcategories of the category of completely regular ordered spaces, analogous to the chain given by the category of $k$-compact spaces, for the different cardinals $k$.
\end{abstract}

0. Introduction. Nachbin [15] has introduced the concept of completely regular ordered space as a generalization of completely regular topological space and, as a generalization of the Stone-Čech compactification, has shown that the category of compact ordered spaces and continuous isotones is an extensive subcategory (2.2) of the category of completely regular ordered spaces and continuous isotones. Herrlich [8] has introduced $k$-compact spaces for each infinite cardinal and has shown that they form a chain of extensive subcategories of the category of completely regular spaces and continuous maps.

Introducing $o$-completely regular filters on a completely regular ordered space, it is shown that for a completely regular ordered space $X$, Nachbin compactification of $X$ is the strict extension of $X$ with all maximal $o$-completely regular filters as the filter trace. Using this fact, we define $k$-compact ordered spaces for every infinite cardinal $k$, and show that categories of $k$-compact ordered spaces are extensive in the category of completely regular ordered spaces and continuous isotones.

It is known [11] that a completely regular space is $k$-compact iff it is $k$-closed (3.2) in its Stone-Čech compactification and $\boldsymbol{N}_{1}$-compact spaces are precisely realcompact spaces, or closed subspaces of powers of the real line $R$.

In our case, $k$-compact ordered spaces are precisely those spaces which are $k$-closed in their Nachbin compactifications, but it is shown that $\boldsymbol{N}_{1}$-compact ordered space need not be an $\boldsymbol{R}$-compact ordered space, i.e. a closed subspace of a power of $R$.

Finally it is noted that by replacing continuous isotones between topological partially ordered spaces with continuous homomorphisms 
between topological lattices, we have the same results in the category of completely regular topological lattices and continuous homomorphisms as those in the category of completely regular ordered spaces.

For general categorical background and terminology we refer to [10] and for topological partially ordered spaces to $[15,16]$.

In the following, every subcategory of a category will be assumed to be full and isomorphism-closed.

\section{Completely regular ordered spaces.}

Definition 1.1. Let $(X, 0)$ be a topological space endowed with a partial order $\leqq$. The partial order $\leqq$ is called continuous provided that whenever $x \notin y$ in $X$, there are open sets $U$ and $V, x \in U$ and $y \in V$, such that if $u \in U$ and $v \in V$, then $u \nsubseteq v$. In this case $(X, \leqq, 0)$ will be called topological partially ordered space.

The category of topological partially ordered spaces and continuous isotones will be denoted by TPOS. It is known [13] that for any family $\left(X_{i}\right)_{i \in I}$ in TPOS and any family $\left(f_{i}: X \rightarrow X_{i}\right)_{i \in I}$ of maps which separates points of $X, X$ can be endowed with a partial order and a topology such that $\left(f_{i}\right)_{i \in I}$ is initial in TPOS. Hence TPOS has products and equalizers so that it is complete.

Definition 1.2. A topological partially ordered space $(X, \leqq, 0)$ will be called completely separated provided that whenever $x \notin y$ in $X$, there exists a continuous isotone $f: X \rightarrow[0,1]$ such that $f(x)>f(y)$, where $[0,1]$ is the unit interval with the usual order and topology.

The following definition is due to Nachbin [15].

Definition 1.3. A completely separated topological partially ordered space $(X, \leqq, 0)$ is said to be a completely regular ordered space if for any point $x$ in $X$ and for any open neighborhood $V$ of $x$ there exists a continuous isotone $f: X \rightarrow I$ and a continuous anti-isotone $g: X \rightarrow I$ such that $f(x)=1=g(x)$ and $\mathbf{C} V \subseteq f^{-1}(0) \cup g^{-1}(0)$, where $I$ denotes the unit interval $[0,1]$ and $\mathbf{C} V$ denotes the complement of $V$ in $X$.

The category of completely regular ordered spaces and continuous isotones will be denoted by CROS.

The proof of the following proposition is quite similar to the case of topological spaces and we will omit it.

Proposition 1.4. For any completely separated topological partially ordered space $(X, \leqq, 0)$, the following are equivalent: 
(1) $(X, \leqq, 0)$ is a completely regular ordered space.

(2) For any point $x$ of $X$ and any open neighborhood $V$ of $x$, there exist finitely many continuous isotones $f_{1}, \cdots, f_{n}: X \rightarrow[-1,1]$ such that $f_{i}(x)=0$ for each $i=1, \cdots, n$ and $\mathbf{C} V \subseteq \cup f_{i}^{-1}(\{-1,1\})$, where $[-1,1]$ is endowed with the usual order and topology.

(3) $\left\{f^{-1}(0) \mid f \in \operatorname{hom}(X, R)\right\}$ forms a subbase for closed sets of $\mathbf{0}$, where $\operatorname{hom}(X, R)$ is the hom-set of CROS and $R$ denotes the real line with the usual order and topology.

(4) $(X, \leqq, 0)$ is an $R$-regular ordered space, i.e. it is isomorphic with a subspace of a power of $R$.

(5) $(X, \leqq, 0)$ is an I-regular ordered space.

COROLlARY 1.5. The category CROS is complete and CROS is an epireflective subcategory of the category TPOS.

Corollary 1.6. A topological partially ordered space $X$ is completely regular iff it is isomorphic with a subspace of a compact ordered space, and $X$ is compact iff it is isomorphic with a closed subspace of a power of the unit interval.

Definition 1.7. Let $X$ be a completely regular ordered space. A filter $\mathscr{F}$ on $X$ is said to be $o$-completely regular if $\mathscr{F}$ has an open base $\mathscr{B}$ satisfying that for each $U \in \mathscr{B}$, there exists a $V \in \mathscr{B}$ with $V \subseteq U$ and there exist finitely many continuous isotones $f_{1}, \cdots, f_{n}: X \rightarrow[-1,1]$ such that $f_{i}(V)=0$ for each $i=1, \cdots, n$ and $\mathbf{C U}$ is contained in $\cup f_{1}^{-1}(\{-1,1\})$.

By a maximal o-completely regular filter on $X$ is meant an $o$ completely regular filter not contained in any other $o$-completely regular filter.

REMARK. For every $o$-completely regular filter, there exists by Zorn's Lemma, a maximal $o$-completely regular filter containing it. In particular, an $o$-completely regular filter $\mathscr{F}$ on a completely regular ordered space $X$ is a maximal $o$-completely regular filter iff for any pair of open sets $U$ and $V$ with $V \subseteq U$ and finitely many continuous isotones $f_{1}, \cdots, f_{n}: X \rightarrow[-1,1]$ such that $f_{i}(V)=0$ for $i=1, \cdots, n$ and $\mathbf{C} U \subseteq$ $\cup f_{1}^{-1}(\{-1,1\})$, either $U \in \mathscr{F}$ or $U \notin \mathscr{F}$ and there exists some $F \in \mathscr{F}$ with $F \cap V=\varnothing$.

THEOREM 1.8. A filter $\mathcal{U}$ on a completely regular ordered space $X$ contains a maximal o-completely regular filter iff $f(\mathcal{U})$ is convergent for each continuous isotone $f: X \rightarrow[-1,1]$. 
Proof. Since a filter containing a convergent filter is again convergent, it is enough to show that every maximal $o$-completely regular filter satisfies the necessary condition. Let $\mathscr{F}$ be a maximal $o$-completely regular filter on $X$ and $f$ a member of $\operatorname{hom}(X,[-1,1])$. Since $[-1,1]$ is compact, $\cap\{\mathrm{cl} f(F) \mid F \in \mathscr{F}\} \neq \varnothing$, where cl is the closure operator on $[-1,1]$. Using the above Remark, it is easy to show that $\cap\{\mathrm{cl} f(F) \mid F \in$ $\mathscr{F}\}$ is a singleton set and $f(\mathscr{F})$ converges to the point.

Conversely, let $\mathcal{U}$ be a filter on $X$ such that for any $f \in$ $\operatorname{hom}(X,[-1,1]) f(\mathcal{U})$ is convergent. Let $x_{f}=\lim f(\mathcal{U})$. Then $f^{-1}\left(\mathbf{0}\left(x_{f}\right)\right) \subseteq$ $U$, where $0\left(x_{f}\right)$ is the neighborhood filter of $x_{f}$. Hence $\cup\left\{f^{-1}\left(0\left(x_{f}\right)\right) \mid f \in\right.$ $\operatorname{hom}(X,[-1,1])\}$ generates a filter; let $\mathscr{F}=\vee f^{-1}\left(0\left(x_{f}\right)\right)$ $(f \in \operatorname{hom}(X,[-1,1]))$. It is easy to show that a join of $o$-completely regular filters is again $o$-completely regular and that $f^{-1}\left(\mathbf{0}\left(x_{f}\right)\right)$ is an $o$-completely regular filter base. Hence $\mathscr{F}$ is an $o$-completely regular filter. Using the above Remark, $\mathscr{F}$ is a maximal $o$-completely regular filter contained in $\mathcal{u}$.

REMARK. For à maximal $o$-completely regular filter $\mathscr{F}$ on a completely regular ordered space $X$ and $f \in \operatorname{hom}(X,[-1,1])$, let $x_{f}=$ $\lim f(\mathscr{F})$. Then $\mathscr{F}=\vee\left\{f^{-1}\left(\mathbf{0}\left(x_{f}\right)\right) \mid f \in \operatorname{hom}(X,[-1,1])\right\}$.

By the definition of completely regular ordered spaces and the above theorem, we have,

COROLlARY 1.9. Every neighborhood filter of a completely regular ordered space is a maximal o-completely regular filter.

COROLlaRY 1.10. A filter $\mathscr{F}$ on a completely regular ordered space $X$ is a minimal Cauchy filter with respect to the uniform structure $U$ generated by $\operatorname{hom}(X, I)$, where $I$ is the interval $[-1,1]$ endowed with the usual uniform structure, iff it is a maximal o-completely regular filter on $X$.

\section{Compact ordered spaces.}

THEOREM 2.1. For a completely regular ordered space $X$, the following are equivalent:

(1) $X$ is compact.

(2) Every o-completely regular filter on $X$ has a cluster point.

(3) Every maximal o-completely regular filter on $X$ is convergent.

Proof. $\quad(1) \Rightarrow(2)$ Trivial.

(2) $\Rightarrow$ (3) Noting that every neighborhood filter on $X$ is a maximal $o$-completely regular filter, it is immediate. 
(3) $\Rightarrow$ (1) Let $U$ be an ultrafilter on $X$. For any $h \in$ $\operatorname{hom}(X,[-1,1]), h(\mathcal{U})$ is convergent, for $[-1,1]$ is compact. Hence $U$ contains a maximal $o$-completely regular filter on $X ; \mathcal{U}$ is convergent.

Definition 2.2. Let $A$ be a subcategory of TPOS. A subcategory $B$ of $A$ is called extensive in $A$ if $B$ is reflective in $A$ and every reflection is a dense embedding.

The following is well known [15], or one can prove it by Corollary 1.6.

THEOREM 2.3. The category COMPOS of compact ordered spaces is extensive in the category CROS.

For the further development, we will characterize the reflection of a completely regular ordered space $X$ by $o$-completely regular filters on $X$.

For a completely regular ordered space $(X, \leqq, 0)$, let $\beta_{0} X$ be the set of all maximal $o$-completely regular filters on $X$, endowed with the topology $0^{*}$ generated by $\left\{U^{*} \mid U^{*}=\left\{\mathcal{M} \in \beta_{0} X \mid U \in \mathcal{M}\right\}, U\right.$ is an open set of $X\}$ and a relation $\leqq$ defined as follows: $\mathcal{M} \leqq \mathcal{N}$ in $\beta_{0} X$ if $\lim f(\mathcal{M}) \leqq \lim f(\mathcal{N})$ for all $f \in \operatorname{hom}(X,[-1,1])$. It is obvious that $\left(\beta_{0} X, \leqq\right)$ is a partially ordered set and that $\left\{U^{*} \mid U \in \mathbf{0}\right\}$ forms a base for 0*. $^{*}$.

Let $\beta_{0}: X \rightarrow \beta_{0} X$ be a map defined by $\beta_{0}(x)=\mathbf{0}(x)$ for $x \in X$. By the construction of $\beta_{0} X, \beta_{0} X$ is precisely the strict extension [1] of $X$ with all maximal $o$-completely regular filters of $X$ as the filter trace. Furthermore for any $x \in X$ and any $f \in \operatorname{hom}(X,[-1,1]), \lim f(0(x))=f(x)$ and $X$ is completely regular; it follows that $\beta_{0}$ is an order isomorphism. Consequently the map $\beta_{0}: X \rightarrow \beta_{0} X$ is a dense embedding.

LEMmA 2.4. The space $\left(\beta_{0} X, \leqq, 0^{*}\right)$ is a topological partially ordered space.

Proof. For any $\mathcal{N} \not \mathcal{M}$ in $\beta_{0} X$, there is $f \in \operatorname{hom}(X,[-1,1])$ with $\lim f(\mathcal{M})<\lim f(\mathcal{N})$. Let $r_{1}$ and $r_{2}$ be elements of $[-1,1]$ with $\lim f(\mathcal{M})<$ $r_{1}<r_{2}<\lim f(\mathcal{N})$ and let $U=f^{-1}\left(\left[-1, r_{1}[)\right.\right.$ and $\left.\left.V=f^{-1}(] r_{2}, 1\right]\right)$. Then it is obvious that $U^{*}$ and $V^{*}$ are neighborhoods of $\mathcal{M}$ and $\mathcal{N}$ respectively and that for any $\mathscr{S} \in V^{*}$ and any $\mathscr{T} \in U^{*}, \mathscr{S} \not \mathscr{T}$.

Definition 2.5. Let $A$ be a category and $C$ an object of $A$. An $A$-morphism $f: A \rightarrow B$ is said to be $C$-extendable if for any $A$ morphism $g: A \rightarrow C$, there is an $A$-morphism $\bar{g}: B \rightarrow C$ with $g=$ $\bar{g} f$. For a class $C$ of $A$-objects, a morphism is said to be $C$-extendable if for any $C \in C$, it is $C$-extendable. 
Lemma 2.6. The morphism $\beta_{0}: X \rightarrow \beta_{0} X$ is $[-1,1]$-extendable in TPOS.

Proof. For any $f: X \rightarrow[-1,1]$ in TPOS and for any $\mathcal{M} \in \beta_{0} X$, define $\bar{f}(\mathscr{M})=\lim f(\mathscr{M})$. It is obvious that $\bar{f}$ is an isotone. Furthermore $\bar{f}$ is continuous, for $\mathcal{M}$ is the trace filter on $X$ of $\mathcal{M}$ itself, $\lim f(\mathcal{M})=\bar{f}(\mathcal{M})$, and $[-1,1]$ is a regular topological space (see [3]).

THEOREM 2.7. The space $\left(\beta_{0} X, \leqq, 0^{*}\right)$ is a compact ordered space.

Proof. First, we will show that $\beta_{0} X$ is a completely regular ordered space. For any $\mathcal{M} \not \mathcal{N}$ in $\beta_{0} X$, there is $f \in \operatorname{hom}(X,[-1,1])$ such that $\lim f(\mathcal{N})<\lim f(\mathcal{M})$. Let $\bar{f}: \beta_{0} X \rightarrow[-1,1]$ be the extension of $f$ determined by Lemma 2.6. Then $\bar{f}(\mathcal{N})<\bar{f}(\mathcal{M})$ and hence $\beta_{0} X$ is completely separated. Let $U^{*}$ be a basic open neighborhood of $M \in \beta_{0} X$. Since $U \in \mathcal{M}$, there is an open set $V \in \mathcal{M}$ with $V \subseteq U$ and there are continuous isotones $f_{1}, \cdots, f_{n}: X \rightarrow[-1,1]$ such that $f_{i}(V)=0$ for each $i$ and $\mathbf{C} U \subseteq$ $\cup\left\{f_{l}^{-1}(\{-1,1\}) \mid 1 \leqq i \leqq n\right\}$. Let $\bar{f}_{l}: \beta_{0} X \rightarrow[-1,1]$ be the extension of $f_{l}$ for each $i$. Then it is obvious that $\bar{f}_{l}(\mathscr{M})=0$ for each $i$ and $\mathbf{C} U^{*} \subseteq$ $\cup\left\{\bar{f}_{l}^{-1}(\{-1,1\}) \mid 1 \leqq i \leqq n\right\}$.

By Theorem 2.1, it is now enough to show that every maximal $o$-completely regular filter on $\beta_{0} X$ is convergent. Let $\mathscr{F}$ be a maximal $o$-completely regular filter on $\beta_{0} X$ and let $\mathscr{B}=\{U \mid U$ : open in $X$ and $\left.U^{*} \in \mathscr{F}\right\}$. Using the fact that $\beta_{0}$ is a $[-1,1]$-extendable extension, we can conclude that $\mathscr{B}$ generates a maximal $o$-completely regular filter, say $\mathcal{M}$ and that $\mathscr{F}$ converges to $\mathcal{M}$. This completes the proof.

THEOREM 2.8. For any completely regular ordered space $X, \beta_{0} X$ is a $[-1,1]$-extendable compactification of $X$. Furthermore, COMPOS is an extensive subcategory of CROS.

Proof. Since every compact ordered space is isomorphic with a closed subspace of a power of $[-1,1]$, a dense map in TPOS is $[-1,1]$-extendable iff it is COMPOS-extendable. Hence for any completely regular ordered space $X, \beta_{0}: X \rightarrow \beta_{0} X$ is the COMPOSreflection of $X$ by Lemma 2.6 and Theorem 2.7.

REMARK 2.9. (1) It is known that the completion of a uniform space $Y$ is the strict extension of $Y$ with all minimal Cauchy filters as the filter trace. Since $\beta_{0} X$ of a completely regular ordered space $X$ is also the strict extension of $X$ with all maximal $o$-completely regular filters on $X$ as the filter trace, by Corollary 1.10 , we can conclude that $\beta_{0} X$ is homeomorphic with the completion of the uniform space $(X, \mathcal{U})$, where $\mathcal{U}$ is generated by $\operatorname{hom}(X,[-1,1])$. 
(2) We define a zero-dimensional ordered space $(X, \leqq, 0)$ as a topological partially ordered space satisfying the following two conditions:

1. For every point $x$ of $X$ and an open neighborhood $V$ of $x$, there exist finitely many continuous isotones $f_{1}, \cdots, f_{n}: X \rightarrow 3$ such that $f_{i}(x)=$ 0 for each $i$ and $\mathbf{C} V \subseteq \cup\left\{f_{i}^{-1}(\{-1,1\}) \mid 1 \leqq i \leqq n\right\}$, where 3 is the chain of three elements $\{-1,0,1\}$ with the usual order and the discrete topology.

2. For $x \notin y$ in $X$, there exists a continuous isotone $f: X \rightarrow 3$ with $f(y)<f(x)$.

Let $Z O S$ denote the category of zero-dimensional ordered spaces and continuous isotones. Then $Z O S$ is an epireflective subcategory of TPOS. Let ZCOMPOS denote the category of compact zerodimensional ordered spaces and continuous isotones. Replacing $[-1,1]$ with 3 in the above arguments, one can easily conclude that ZCOMPOS is an extensive subcategory of $Z O S$.

\section{3. $\quad k$-compact ordered spaces.}

Definition 3.1. Let $k$ be an infinite cardinal. A completely regular ordered space $X$ is called $k$-compact if every maximal $o$-completely regular filter on $X$ with the $k$-intersection property is convergent.

Definition 3.2. Let $k$ be an infinite cardinal, and let $X$ be a topological space. A subset of $X$ is called a $G_{k}$-set if it is an intersection of fewer than $k$ open subsets of $X$. A subset of $X$ is called $k$-closed if it is closed with respect to the topology generated by all $G_{k}$-sets of $X$.

LEMMA 3.3. A completely regular ordered space $X$ is $k$-compact iff it is $k$-closed in $\beta_{0} X$.

Proof. Noting that $\beta_{0} X$ is the strict extension of $X$ with all maximal $o$-completely regular filters as the filter trace, the proof is immediate from Proposition 2.4 [11].

By Remark 2.9(1) and Lemma 3.2 [11], one has

COROllary 3.4. A completely regular ordered space $X$ is $k$ compact iff it is $k$-complete with respect to the uniform structure $U$ on $X$ generated by $\operatorname{hom}(X,[-1,1])$, i.e. every $U$-Cauchy filter with the $k$ intersection property is convergent.

For an infinite cardinal $k$, the category of $k$-compact ordered spaces and continuous isotones will be denoted by $k C O S$. 
For a subcategory $A$ of TPOS, let $B$ be an extensive subcategory of $A$. For any infinite cardinal $k$, let $B_{k}$ denote the subcategory of $A$ determined by those members of $A$ which are $k$-closed in their $B$ reflections. By replacing continuous maps in the proof of Theorem 2.2 [11] with continuous isotones, one has the following

THEOREM 3.5. If $A$ is hereditary, then $B_{k}$ is also an extensive subcategory of $A$.

Using Lemma 3.3 and the fact that COMPOS is extensive in the hereditary category CROS, we have the following

THEOREM 3.6. The category $k C O S$ is extensive in CROS.

COROLlaRy 3.7. The category $k C O S$ is complete, and every $k$ closed subspace of a $k$-compact ordered space is again a $k$-compact ordered space.

Definition 3.8. Let $k$ be an infinite cardinal. A Hausdorff space is said to be $k$-Lindelöf if every filter with the $k$-intersection property has a cluster point.

We note that $\boldsymbol{\aleph}_{0}$-Lindelöf spaces are exactly compact spaces and that $\boldsymbol{\aleph}_{1}$-Lindelöf spaces are exactly Lindelöf spaces.

PROPOSITION 3.9. A $k$-Lindelöf completely regular ordered space $X$ is a $k$-compact ordered space.

Proof. For any maximal $o$-completely regular filter $\mathscr{F}$ on $X$ with the $k$-intersection property, let $x$ be a cluster point of $\mathscr{F}$. Then $\mathbf{0}(x) \vee \mathscr{F}$ exists; $\mathbf{0}(x)=\mathscr{F}$. Hence $\mathscr{F}$ is convergent.

PROPOSITION 3.10. If a filter $\mathscr{F}$ on a completely regular ordered space $X$ contains a maximal o-completely regular filter with the countable intersection property, then $f(\mathscr{F})$ is convergent for any continuous isotone $f: X \rightarrow R$.

Proof. It is enough to show that for any maximal $o$-completely regular filter $\mathscr{F}$ with the countable intersection property and a continuous isotone $f: X \rightarrow R, f(\mathscr{F})$ is convergent. Since $f(\mathscr{F})$ is a filter base with the countable intersection property and $R$ is Lindelöf, $f(\mathscr{F})$ has a cluster point. Moreover, by the same argument as that in the proof of Theorem 1.8 , one can easily show that $f(\mathscr{F})$ has only one cluster point and that $f(\mathscr{F})$ converges to the point. 
REMARK. It is known [12] that for a filter $\mathscr{F}$ on a completely regular space $X$, and for any continuous map $f: X \rightarrow R, f(\mathscr{F})$ is convergent iff $\mathscr{F}$ contains a maximal completely regular filter on $X$ with the countable intersection property. However the converse of the above theorem need not be true (see Remark 3.15).

Definition 3.11. Let $P$ be an object of TPOS. An object $A$ of TPOS is called $P$-compact if $A$ is isomorphic with a closed subspace of a power of $P$.

We note that the subcategory of $P$-compact spaces of TPOS is productive and closed hereditary.

Since $R$ is Lindelöf, $R$ is an $\boldsymbol{N}_{1}$-compact ordered space. Using the fact that $\boldsymbol{N}_{1} C O S$ is productive and closed hereditary, one has,

Proposition 3.12. Every $R$-compact ordered space is an $\boldsymbol{\aleph}_{1}$ compact ordered space.

It is well known [8] that $R$-compact topological spaces are exactly $\boldsymbol{N}_{1}$-compact topological spaces. But the converse of the above theorem does not hold (see Remark 3.15).

Definition 3.13. A completely regular ordered space $X$ is said to be $o$-pseudocompact if for each continuous isotone $f: X \rightarrow R, f(X)$ is bounded.

REMARK. A completely regular ordered space $X$ is compact iff it is an $\boldsymbol{o}$-pseudocompact, $R$-compact ordered space.

EXAMPLE 3.14. Let $E=\{-\infty\} \cup R \cup\{\infty\}$ endowed with the usual order and a topology generated by $\{\{-\infty\}\} \cup\{\{\infty\}\} \cup\{\boldsymbol{0}(R)\}$, where $\mathbf{0}(R)$ is the usual topology on $R$. Then it is obvious that $E$ is not an $R$-compact ordered space, but an $\boldsymbol{N}_{1}$-compact ordered space, for $E$ is not compact but $o$-pseudocompact. We note that $E$ is not pseudocompact.

Remark 3.15. The above example shows that

(1) The category of $R$-compact ordered spaces is a proper subcategory of $\boldsymbol{N}_{1} C O S$, and

(2) The converse of Proposition 3.10 does not hold.

For an infinite cardinal $k$, let $P_{k^{+}}=I^{k}-\{(0)\}$, where $k^{+}$is the successor of $k, I$ denotes the interval $[-1,1],(0)$ is the element of $I^{k}$ whose every coordinate is 0 , and $P_{k^{+}}$is the subspace of $I^{k}$ and for a limit 
cardinal $k>\boldsymbol{N}_{0}$, let $P_{k}=\Pi_{t<k} P_{t^{+}}$. By the exactly same arguments as those in [14], one has,

THEOREM 3.16. For any infinite cardinal $k>\boldsymbol{N}_{0}$, a completely regular ordered space $X$ is $k$-compact iff it is a $P_{k}$-compact ordered space. For $k=\boldsymbol{\aleph}_{0}, X$ is $\boldsymbol{\aleph}_{0}$-compact iff it is an I-compact ordered space.

\section{Concluding remarks.}

4.1. Every completely regular space is precisely completely regular ordered space endowed with the discrete order, and every (maximal) completely regular filter on a completely regular space $X$ is also (maximal resp.) $o$-completely regular filter on the associated completely regular ordered space of $X$; the Stone-Čech compactification of $X$ is nothing but the Nachbin compactification of the associated completely regular ordered space of $X$. Furthermore a completely regular space $X$ is $k$-compact in the sense of Herrlich [8] iff the associated completely regular ordered space is a $k$-compact ordered space.

4.2. If one defines an $o$-zero-dimensional filter $\mathscr{F}$ on a zerodimensional ordered space $X$ by replacing $[-1,1]$ with 3 in Definition 1.7 , one can also define the $o$-zero-dimensionally $k$-compact ordered spaces, i.e. a zero-dimensional ordered space $X$ is $o$-zero-dimensionally $k$-compact iff every maximal $o$-zero-dimensional filter on $X$ with the $k$-intersection property is convergent. By the same argument as above, the category $k Z C O M P O S$ of $o$-zero-dimensionally $k$-compact ordered spaces is also an extensive subcategory of $Z O S$ for each infinite cardinal $k$.

4.3. A topological lattice $L$ will be called completely regular if for any point $x$ of $L$ and an open neighborhood $V$ of $x$, there exist finitely many continuous homomorphisms $f_{1}, \cdots, f_{n}: L \rightarrow[-1,1]$ such that $f_{i}(x)=0$ for each $i=1, \cdots, n$ and $\left.\mathbf{C} V \subseteq \cup f_{i}^{-1}(\{-1,1\}) \mid 1 \leqq i \leqq n\right\}$. It is immediate that a topological lattice $L$ is completely regular iff it is isomorphic with a sublattice of a power of $[-1,1]$; hence every completely regular topological lattice is distributive, and contrary to the case of compact ordered spaces, there is a compact topological lattice which is not completely regular.

However, a completely regular topological lattice is compact iff it is isomorphic with a closed sublattice of a power of $[-1,1]$; the category of $I$-COMPTL of compact completely regular topological lattices is an extensive subcategory of the category CRTL of completely regular topological lattices and continuous homomorphisms. If one defines a (maximal) 1-completely regular filter on $X \in C R T L$ by replacing con- 
tinuous isotones in Definition 1.7 with continuous homomorphisms, the 1-completely regular filters enjoy the corresponding results obtained for $o$-completely regular filters.

Furthermore, for $(L, \vee, \wedge, 0) \in C R T L$, its $I$-COMPTL-reflection is given as follows: its underlying topological space is the strict extension of $(L, 0)$ with all 1-maximal completely regular filters as the filter trace, and for a pair $(\mathcal{M}, \mathcal{N})$ of 1 -maximal completely regular filters on $L, \mathcal{M} \vee \mathcal{N}$ is the filter generated by $\left\{M \vee_{L} N \mid M \in M, N \in \mathcal{N}\right\}$, and $\mathcal{M} \wedge \mathcal{N}$ is the filter generated by $\left\{M \wedge_{L} N \mid M \in \mathcal{M}, N \in \mathcal{N}\right\}$. Let us denote the space by $\left(\beta_{1} L, \vee, \wedge, 0^{*}\right)$ and $\beta_{1}: L \rightarrow \beta_{1} L$ defined by $\beta_{1}(x)=0(x)$ for each $x \in X$. Then $\beta_{1}: L \rightarrow \beta_{1} L$ is the I-COMPTL-reflection of $L$.

Definition 4.4. A completely regular topological lattice $L$ is called $k$-compact for an infinite cardinal if every maximal 1-completely regular filter on $L$ with the $k$-intersection property is convergent.

RemarK. A completely regular topological lattice $L$ is $k$-compact iff it is $k$-closed in $\beta_{1} L$.

Using Theorem 1.7 and Proposition 2.3 [4], one has immediately the follwoing

THEOREM 4.5. The category of $k$-compact topological lattices is extensive in the category CRTL.

REMARK 4.6. Example 3.14 also shows that $\boldsymbol{\aleph}_{1}$-compact topological lattice need not be isomorphic with a closed sublattice of a power of $R$.

4.7. The problem to characterize $R$-compact ordered spaces and $R$-compact topological lattices, remains unsolved.

\section{REFERENCES}

1. B. Banaschewski, Extensions of topological spaces, Canad. Math. Bull., 7 (1964), 1-22.

2. G. Birkhoff, Lattice Theory, 3rd ed. Amer. Math. Soc., Providence (1967).

3. N. Bourbaki, Topologie générale, Hermann, Paris (1960).

4. T. H. Choe and Y. H. Hong, Extensive subcategories in universal topological algebras, (preprint).

5. O. Garcia, Partially ordered topological spaces, Doct. Dissertation, McMaster University, Hamilton (1972).

6. L. Gillman and M. Jerrison, Rings of Continuous Functions, D. Van Nostrand Co., Princeton (1960)

7. H. Herrlich, $\mathscr{E}$-kompakte Räume, Math. Z., 96 (1967), 228-255.

8. - Fortsetzbarkeit stetiger Abbildungen und Kompaktheitgrad topologischer Räume, Math.

Z., 96 (1967), 64-72.

9. - Topologische Reflexionen und Coreflexionen, Springer, Lect. Notes in Math., 78 (1968).

10. H. Herrlich and G. E. Strecker, Category Theory, Allyn and Bacon Inc., Boston (1973). 
11. S. S. Hong, On k-compactlike spaces and reflective subcategories, General Topology and Appl., 3 (1973), 319-330.

12. S. S. Hong and L. D. Nel, E-compact convergence spaces and E-filters, Kyungpook Math. J., 15 (1975), 183-189.

13. Y. H. Hong, Studies on categories of universal topological algebras, Doct. Dissertation, McMaster University, Hamilton, (1974).

14. M. Hušek, The class of $k$-compact spaces is simple, Math. Z., 110 (1969), 123-126.

15. L. Nachbin, Topology and Order, D. Van Nostrand Co., Princeton (1965).

16. L. E. Ward Jr., Partially ordered topological spaces, Proc. Amer. Math. Soc., (1954), 144-161.

Received October 23, 1975 and in revised form April 29, 1976.

MCMASTER UNIVERSITY,

AND

SOOKMYUNG WOMEN'S UNIVERSITY,

Seoul, Korea 



\section{Pacific Journal of Mathematics}

\section{Vol. 66, No. $1 \quad$ November, 1976}

Helen Elizabeth. Adams, Factorization-prime ideals in integral domains ............ Patrick Robert Ahern and Robert Bruce Schneider, The boundary behavior of Henkin's kernel.

Daniel D. Anderson, Jacob R. Matijevic and Warren Douglas Nichols, The Krull

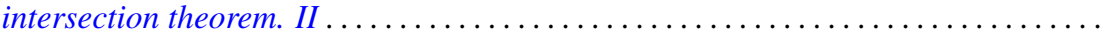

Efraim Pacillas Armendariz, On semiprime P.I.-algebras over commutative regular

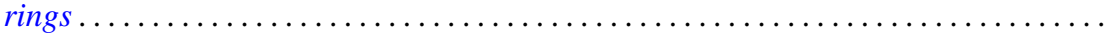

Robert H. Bird and Charles John Parry, Integral bases for bicyclic biquadratic fields

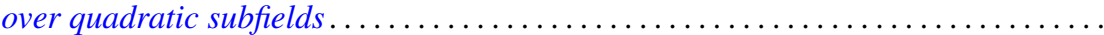

Tae Ho Choe and Young Hee Hong, Extensions of completely regular ordered

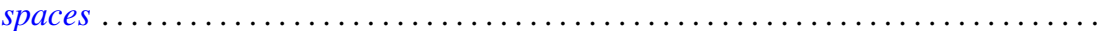

John Dauns, Generalized monoform and quasi injective modules ...............

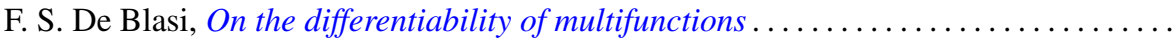

Paul M. Eakin, Jr. and Avinash Madhav Sathaye, R-endomorphisms of $R[[X]]$ are

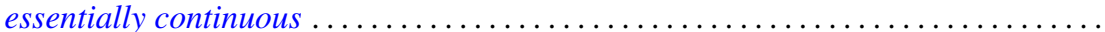

Larry Quin Eifler, Open mapping theorems for probability measures on metric

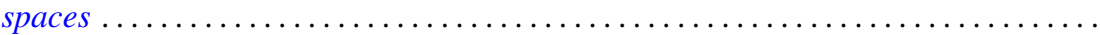

Garret J. Etgen and James Pawlowski, Oscillation criteria for second order self adjoint

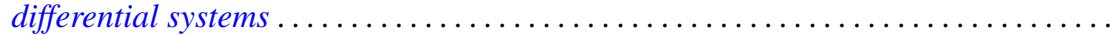

Ronald Fintushel, Local $S^{1}$ actions on 3-manifolds .

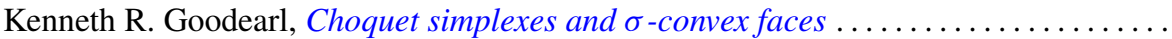

John R. Graef, Some nonoscillation criteria for higher order nonlinear differential

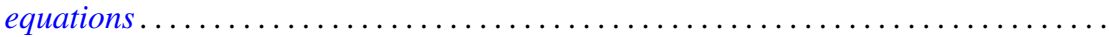

Charles Henry Heiberg, Norms of powers of absolutely convergent Fourier series: an example.

Les Andrew Karlovitz, Existence of fixed points of nonexpansive mappings in a space

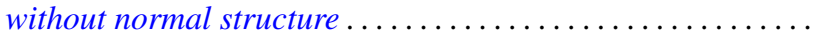

Gangaram S. Ladde, Systems of functional differential inequalities and functional

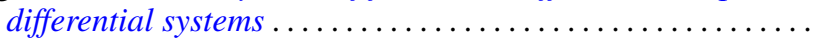

Joseph Michael Lambert, Conditions for simultaneous approximation and interpolation with norm preservation in $C[a, b]$.

Ernest Paul Lane, Insertion of a continuous function.

Robert F. Lax, Weierstrass points of products of Riemann surfaces .

Dan McCord, An estimate of the Nielsen number and an example concerning the Lefschetz fixed point theorem...

Paul Milnes and John Sydney Pym, Counterexample in the theory of continuous functions on topological groups...

Peter Johanna I. M. De Paepe, Homomorphism spaces of algebras of holomorphic functions

Judith Ann Palagallo, A representation of additive functionals on $L^{p}$-spaces,

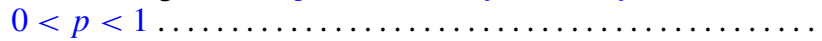

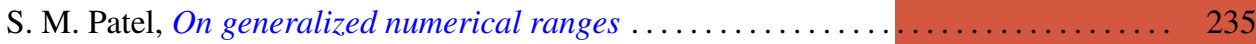

Thomas Thornton Read, A limit-point criterion for expressions with oscillatory

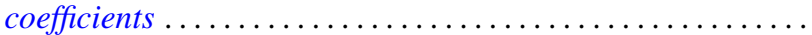

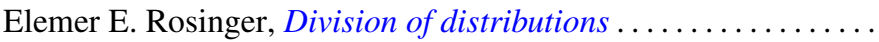

Peter S. Shoenfeld, Highly proximal and generalized almost finite

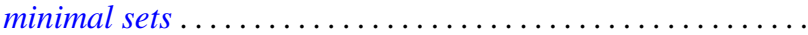

R. Sirois-Dumais and Stephen Willard, Quotient-universal sequential spaces

Robert Charles Thompson, Convex and concave functions of singular values of matrix sums....

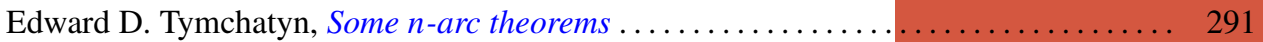

